endonucleases do not detect the relevant sites.

How can the inherent weaknesses of the restriction enzyme approach be overcome? The answer may lie with an unlikelysounding, but recently perfected, technique for sequencing unique stretches of DNA in unfractionated genomic DNA. George Church of Harvard University has used a sophisticated blend of blot hybridization and Maxam-Gilbert ${ }^{19}$ sequencing to locate all 5-methylcytosine residues in a region of the globin gene of transformed mouse cells (see ref. 4). It would now be interesting to use this technique to monitor other $\mathrm{CpG}$ sequences during activation of the vitellogenin genes.

In evaluating recent data on gene expression and DNA methylation, it may be helpful to take an evolutionary perspective, starting from the observation that the level of $\mathrm{CpG}$ methylation is considerably higher in vertebrates than in invertebrates. Apart from the much quoted case of Drosophila, in which methylated cytosine residues have not been detected at all $^{20}$, other invertebrates generally have a minor fraction of heavily methylated DNA in their genome $e^{21}$. No clear example of a methylated gene has yet been detected in invertebrates. Since these animals nevertheless exhibit cellular determination and differentiation, it seems likely that, in animals, DNA methylation was not involved in the evolution of these basic mechanisms.

Why, then, are the majority of vertebrate genes methylated? One possibility is that DNA methylation in invertebrates has a purely suppressive effect - simply being used to condemn sequences to transcriptional silence - while in vertebrates, the spread of methylation has elicited mechanisms that allow genes to tolerate and, in some cases, to exploit methylation for the control of expression.

There are three ways in which a gene might circumvent suppression by DNA methylation. First, key sequences might be maintained in an unmethylated condition by rendering them refractory to methylation, as in the case of genes whose $5^{\prime}$ domains are unmethylated in expressing and non-expressing tissues alike ${ }^{8,9}$. Second, genes may tolerate methylated bases at their regulatory sequences, as the ribosomal RNA genes of Xenopus appear to do. Last, expression of the gene may be suppressed by methylation in inappropriate tissues, but in tissues where the gene is to be expressed transcription can proceed following demethylation. It is this role for methylation that has hitherto attracted most interest, and it is to this category that the globin genes may belong.

A demethylation step is usually envisaged as a pre-requiste for transcription of genes regulated by loss of DNA methylation. A weakness of this view is that to regulate genes separately, a distinct mechanism is required to demethylate each kind of gene - it is difficult to see why this should have evolved. An alternative control mechanism, and one whose evolution is perhaps easier to envisage as an ancestral gene moved from the unmethylated to the methylated condition, is that activation of the gene begins with the gene still in a methylated state. Activation then leads to demethylation which, in turn, relaxes control of the gene. Here, demethylation is not thought of as a mechanism to facilitate initial activation of the gene, but to simplify continued expression once activation has occurred, perhaps by bringing the gene under more 'everyday' control mechanisms.

Although we do not yet know how any eukaryotic gene is regulated, preliminary evidence suggests that the degree of involvement of DNA methylation is variable. This is consistent with the view that methylation of genes was a relatively late occurrence in animal evolution, and it suggests that we should not expect a coherent unified mechanism for control of gene expression based on DNA methylation. In order to learn just how much of gene control is due to modulation of DNA methylation patterns, we must await future studies on a gene-by-gene basis.

Adrian P. Bird is at the MRC Mammalian Genome Unit, King's Buildings, West Mains Road, Edinburgh EH9 $3 J T$.

\title{
Biotechnology
}

\section{Time for plant cell culture?}

\section{from M.W. Fowler}

WITH the announcement last year by Mitsui Petrochemical Industries of a process for the production of the pharmaceutical and dye shikonin by large-scale plant cell culture, plant cell biotechnology at last became a reality on the industrial scene. Although the scale of activity (some 750 litres bioreactor capacity) is small in comparison with large-scale microbial fermentation processes, the shikonin process nonetheless marks an important first step forwards in plant cell biotechnology. Hitherto this area of biotechnology had been considered by many to be 'just another way of looking at plant biochemistry' rather than as a genuine alternative way of manufacturing plant natural products.

Plant cell culture may prove of particular value in the future in situations where a new chemical structure with interesting properties has been identified but the source plant is particularly difficult to grow. Of course, the possibility of deriving the compound by organic synthesis must be considered first - but there again there are now several reports of the synthesis by cell cultures of chemical structures that are quite unique. Before cell culture sets off in new directions, however, the technology must first be proved on an existing product.

In Japan, the Japanese Salt and Tobacco Monopoly has made particular progress towards the development of a tobacco biomass system and has used conventional stirred tank bioreactors (fermenters) at volumes in excess of 6,000 litres. Pharmaceuticals have been the target in West German laboratories and substantial success has been achieved at the University of Tübingen in the development of a process for the biotransformation of lowvalue digitoxin to the important and highvalue cardiatonic digoxin.

Plant cell cultures are maintained in liquid culture in much the same way as yeasts and other microbial cells, but there are some major differences: plant cells grow much more slowly than microbial cells and doubling times of the order of 20-150 h are typical. Sub-culture periods are thus much longer. Also, it is easy to maintain microbial species in a culture bank, either lyophilized or cryopreserved, but similar techniques are only just being developed for plant cell cultures. In consequence, large liquid culture banks are needed to support plant cell cultures. Even if cell preservation techniques do become viable, the long time required to grow up plant cell cultures still means that careful planning is essential to initiate large-scale cultures.

On present-day costings, plant cell culture is likely to be considered only for high-value low-market-volume products. In general, products have to have a whole sale value of at least $\$ 250-500$ per $\mathrm{kg}$ to make them worth considering as targets for a cell culture process. Once one or two processes have been developed successfully, however, the increased know-how and confidence are likely to lead to a widening of the target window.

What are likely to be the key developments in the years ahead? The comparatively few large-scale systems that are being actively tested have not encountered major problems. Fairly sophisticated technologies such as fluidized bed and immobilized cell systems are also showing rapid development. One area which is in urgent need of consideration is downstream processing, the unglamorous but often highly expensive end of the business. It is back in the cell however that the biggest problems still face us. Lack of the fundamental biochemical and genetic information that would enable control of product yields is the chief constraint on the development of plant cell biotechnology and there is much fundamental research that needs to be done.

M. W. Fowler is Director of the Wolfson Institute of Biotechnology at the University of Sheffield, Sheffield S10 2TN. 